\title{
Double Points Local Hardy-Littlewood Maximal Operator
}

\author{
Futao Song and Na Ju \\ Mathematics Science College, Harbin Normal University, Harbin 150025, China \\ Correspondence should be addressed to Futao Song; ftsonghrb@163.com
}

Received 3 July 2013; Accepted 7 November 2013

Academic Editor: Ademir Fernando Pazoto

Copyright (C) 2013 F. Song and N. Ju. This is an open access article distributed under the Creative Commons Attribution License, which permits unrestricted use, distribution, and reproduction in any medium, provided the original work is properly cited.

A double points local Hardy-Littlewood maximal operator $M_{\{a, b\}, k, \text { loc }}$ is defined and investigated in Euclidean spaces. It is proved that $M_{\{a, b\}, k, \text { loc }}$ is bounded on $L^{p}(w)$ when $p>1$ and from $L^{1}(w)$ to $L^{1, \infty}(w)$ with weight function $w \in A_{\{a, b\}, k, \text { loc }}$, the class of double points local $A_{p}$ weights which is larger than the Muckenhoupt $A_{p}$ class and the local $A_{p}$ weights defined by Lin and Stempak.

\section{Introduction}

The Hardy-Littlewood maximal operator $M$ is defined on the class of the locally integrable functions on $\mathbb{R}^{d}$ by

$$
M f(x)=\sup _{\mathrm{Q} \ni x} \frac{1}{|Q|} \int_{Q}|f(x)| d x, \quad x \in \mathbb{R}^{d},
$$

where the supremum is taken over all cubes $Q \subset \mathbb{R}^{d}$ containing $x$, with sides parallel to the coordinate axes. It is one of the most fundamental and important operators in Fourier analysis and often used to majorize other important operators. Many papers are devoted to study the HardyLittlewood operator and its generalizations. Li et al. [1] gave the estimates of Hardy-Littlewood operator on the multilinear spaces. Lerner [2] showed that Hardy-Littlewood maximal operator was bounded on variable $L^{p}$ spaces. Gallardo [3] characterized the pairs of weights $(u, w)$ for which the Hardy-Littlewood maximal operator $M$ satisfies a weak type integral inequality.

In this paper we consider $\mathbb{R}^{d}, d \geq 1$, equipped with the metric induced by the norm

$$
\|x\|=\max \left\{\left|x_{1}\right|,\left|x_{2}\right|, \ldots,\left|x_{d}\right|\right\}, \quad x=\left(x_{1}, x_{2}, \ldots, x_{d}\right) .
$$

By a cube we always mean the usual closed cube with sides parallel to the coordinate axes; in terms of the metric, $Q(x, r)=\left\{y \in \mathbb{R}^{d}:\|y-x\| \leq r\right\}$ denotes the cube centered at $x \in \mathbb{R}^{d}$ with "radius" $r>0$. $|E|$ denotes the Lebesgue measure of a measurable set $E \subset \mathbb{R}^{d}$.
For given $a, b \in \mathbb{R}^{d}$ and $k>0$, denote by $\mathcal{O}_{\{a, b\}, k}(y)$ the family of all cubes centered at $y$ with radius $r \leq k \min \{\| y-$ $a\|\| y-b \|$,$\} , and define$

$$
\mathcal{O}_{\{a, b\}, k}=\bigcup_{y \in \mathbb{R}^{d}} \mathcal{O}_{\{a, b\}, k}(y) .
$$

Note that for every $Q \in \mathcal{O}_{\{a, b\}, k}, 0<k<1$, we have $a, b \notin Q$. In particular, $\mathcal{O}_{\{a, b\}, k}=\mathscr{O}_{k}$ when $a$ is the origin and $b$ tends to $\infty$, where $\mathscr{O}_{k}=\bigcup_{y \in \mathbb{R}^{d}} \mathcal{O}_{k}(y)$ and $\mathcal{O}_{k}(y)$ is the collection of all the cubes centered at $y$ with radius $r \leq k\|y\|$. The cubes in $\mathscr{O}_{k}$ are away from the origin, while the ones in $\mathcal{O}_{\{a, b\}, k}$ are away from two points $a$ and $b$.

Given $0<k<1$, we define the (noncentered) double points local Hardy-Littlewood maximal operator

$$
\begin{array}{r}
M_{\{a, b\}, k, \text { loc }} f(x)=\sup _{x \in Q \in \mathcal{O}_{\{a, b\}, k}} \frac{1}{|Q|} \int_{Q}|f(t)| d t, \\
x \in \mathbb{R}^{d} \backslash\{a, b\},
\end{array}
$$

where the supremum is taken over all cubes $Q \in \mathcal{O}_{\{a, b\}, k}$ containing $x$. It generalizes the local maximal operator $M_{k, \text { loc }}$ defined in [4] for locally integrable functions on $\mathbb{R}^{d} \backslash\{0\}$ by

$$
M_{k, \mathrm{loc}} f(x)=\sup _{x \in \mathrm{Q} \in \Theta_{k}} \frac{1}{|Q|} \int_{Q}|f(t)| d t, \quad x \in \mathbb{R}^{d} \backslash\{0\} .
$$

The purpose of this paper is to investigate the double points local Hardy-Littlewood maximal operator. The paper was organized as follows. 
The definition of the class $A_{\{a, b\}, k, \text { loc }}^{p}$ of double points local $A_{p}$ weights is given in Section 2 , as well as the coincidence of $A_{\{a, b\}, k, \text { loc }}^{p}$ for $0<k<1$. That is, $A_{\{a, b\}, k, \text { loc }}^{p}=A_{\{a, b\}, m, \text { loc }}^{p}$ for $0<k, m<1$. Section 3 includes some lemmas that need to prove the main result Theorem 6 given in Section 4 . We prove that for a weight $w$ satisfying the double points local $A_{p}$ condition, the operator $M_{\{a, b\}, k, \text { loc }}$ is bounded on $L^{p}(w)$, $1<p<\infty$ and bounded from $L^{1}(w)$ to $L^{1, \infty}(w)$.

That $w$ is a weight on $\mathbb{R}^{d}$ means that $w$ is a nonnegative locally integrable function and finite almost everywhere. $L^{p}(w)$ and $L^{1, \infty}(w)$ denote the class of weighed $L^{p}$ space and weighed weak $L^{1}$ space, with the norm $\|f\|_{L^{p}(w)}^{p}=$ $\int_{\mathbb{R}^{d}}|f(x)|^{p} w(x) d x$ and $\|f\|_{L^{1, \infty}(w)}=\sup _{t>0}\left(t \int_{|f|>t} w(x) d x\right)$, respectively.

\section{Double Points Local $A_{p}$ Weights}

For a weight $w$ on $\mathbb{R}^{d}$, we say that $w \in A_{p}$ for $1<p<\infty$ if there is a constant $C>0$ such that

$$
\sup _{\mathrm{Q} \subset \mathbb{R}^{d}}\left(\frac{1}{|Q|} \int_{\mathrm{Q}} w(x) d x\right)\left(\frac{1}{|Q|} \int_{\mathrm{Q}} w(x)^{1-p^{\prime}} d x\right)^{p-1} \leq C,
$$

here and below $1 / p+1 / p^{\prime}=1$. We say that $w \in A_{1}$ if there is a constant $C>0$ such that $M w(x) \leq C w(x)$. The smallest constant $C$ is called the $A_{p}$ constant of $w$ and denoted by $\|w\|_{A_{p}}$. The definition of $A_{p}$ weights was first introduced by Muckenhoupt [5] for $d=1$. Muckenhoupt proved the characterization of the weighed type $(p, p)$ for Hardy-Littlewood maximal operator. That is, for $w \in A_{p}$, $p \geq 1$, the operator $M$ is bounded on $L^{p}(w)$ when $p>1$ and bounded from $L^{1}(w)$ into $L^{1, \infty}(w)$ when $p=1$; moreover, we have

$$
\begin{gathered}
\|M f\|_{L^{p}(w)} \leq C\|w\|_{A_{p}}^{p^{\prime}}\|f\|_{L^{p}(w)}, \quad f \in L^{p}(w), p>1, \\
\|M f\|_{L^{1, \infty}}(w) \leq C\|w\|_{A_{1}}\|f\|_{L^{1}(w)}, \quad f \in L^{1}(w),
\end{gathered}
$$

where the constant $C$ is independent of $w$ (cf. [6]).

The class of the local $A_{k \text {,loc }}^{p}$ weights is defined in [4] by considering the cubes in $\mathcal{O}_{k}$ and the corresponding constant is denoted as $\|w\|_{A_{k, \text { loc }}^{p}}$. Lin and Stempak proved that for $w \in A_{k, \text { loc }}^{p}$, the local Hardy-Littlewood maximal operator is bounded from $L^{p}(w)$ to $L^{p}(w)$ when $p>1$ and from $L^{1}(w)$ to $L^{1, \infty}(w)$.

In order to prove the coincidence of the classes $A_{\{a, b\}, k, \text { loc }}^{p}$ for $0<k<1$, we need the following lemma.

Definition 1. Let $0<k<1,1 \leq p<\infty, a, b \in \mathbb{R}^{d}$, and $w \in L_{\text {loc }}\left(\mathbb{R}^{d} \backslash\{a, b\}\right)$ a nonnegative function. If there exists a constant $C>0$ such that

$$
\sup _{Q}\left(\frac{1}{|Q|} \int_{Q} w(x) d x\right)^{1 / p}\left(\frac{1}{|Q|} \int_{Q} w(x)^{-p^{\prime} / p} d x\right)^{1 / p^{\prime}} \leq C
$$

for $1<p<\infty$, where the supremum takes over all the cubes $Q \in \mathcal{O}_{\{a, b\}, k}$, then $w$ is called the double points local $A_{p}$ weight associated with $a$ and $b$. And for $p=1$, the class $A_{\{a, b\}, k, \text { loc }}^{1}$ is defined by

$$
\frac{1}{|Q|} \int_{Q} w(x) d x \leq C \underset{x \in Q}{\operatorname{essinf}} w(x),
$$

for all $Q \in \mathcal{O}_{\{a, b\}, k}$. Denote the collection of all the double points local $A_{p}$ weights as $A_{\{a, b\}, k, \text { loc }}^{p}=A_{\{a, b\}, k, \text { loc }}^{p}\left(\mathbb{R}^{d}\right)$. The smallest constant $C$ is called the double points local $k-A_{p}$ constant and denoted as $\|w\|_{A_{\{a, b\}, k, \text { loc }}^{p}}$.

According to the definition above, it is easy to see that $A_{p} \subset A_{\{a, b\}, k, \text { loc }}^{p}$. Furthermore, if $w \in A_{p}$, then $\|w\|_{A_{\{a, b\}, k, \text { loc }}^{p}} \leq$ $\|w\|_{A_{p}}$. But $A_{p} \neq A_{\{a, b\}, k, \text { loc }}^{p}$. In fact, let $w(x)=\min \{\| x-$ $a\|\| x-b \|$,$\} ; then for any t \in \mathbb{R}$, we have $w(x)^{t} \in A_{\{a, b\}, k, \text { loc' }}^{p}$, while $w(x)^{t} \in A_{p}$ if and only if $-d<t<d(p-1)$ when $p>1$ and $-d<t \leq 0$ when $p=1$.

On the other hand, define $\mathcal{O}_{\{a\}, k}=\bigcup_{y \in \mathbb{R}^{d}} \mathcal{O}_{\{a\}, k}(y)$, where $\mathcal{O}_{\{a\}, k}(y)$ is the collection of the cubes centered at $y$ with radius no more than $k\|x-a\|$. Then the cubes in $\mathcal{O}_{\{a\}, k}$ are just the translations of the cubes in $\mathcal{O}_{k}$ along $a$ mentioned in [4]. Replacing $\mathcal{O}_{\{a, b\}, k}$ with $\mathcal{O}_{\{a\}, k}$ in Definition 1 gives the definition of the class $A_{\{a\}, k, \text { loc }}^{p}$. It is also easy to see that $A_{\{a\}, k, \text { loc }}^{p} \subset A_{\{a, b\}, k, \text { loc }}^{p}$. The same process gives $\mathcal{O}_{\{b\}, k}$ and consequently the class $A_{\{b\}, k, \text { loc }}^{p}$. We also have the relation $A_{\{b\}, k, \mathrm{loc}}^{p} \subset A_{\{a, b\}, k, \mathrm{loc}}^{p}$.

Lemma 2. Let $0<k<1$; for every $0<r<1$, there exists a $n=n(k, r)$ satisfying the following property: if $R$ and $\widehat{R}$ are the cubes centered at $x(\neq a, b)$ with radius $r \min \{\|x-a\|,\|x-b\|\}$ and $(r+k(1-r)) \min \{\|x-a\|,\|x-b\|\}$, respectively, there exist at most $n$ cubes $Q_{j} \subset \widehat{R}$ and $Q_{j} \in \mathcal{O}_{\{a, b\}, k}, 1 \leq j \leq n$, covering $\widehat{R} \backslash R$ and $\left|Q_{j} \cap R\right| \geq C|R|$, where $Q_{j}, 1 \leq j \leq n$ are the cubes with centers located on $\partial R$ and radius $k(1-r) \min \{\|x-a\|,\|x-b\|\}$ and $C=\min \left\{1,(k(1-r) / 2 r)^{d}\right\}$.

Proposition 3. Let $0<k, m<1$, and $1 \leq p<\infty$. Then

$$
A_{\{a, b\}, k, \mathrm{loc}}^{p}=A_{\{a, b\}, m, \mathrm{loc}}^{p}
$$

The proofs of Lemma 2 and Proposition 3 are analogous to the proofs of Lemma 2.1 and Proposition 2.2 in [4], respectively, so we omit them here.

By Proposition 3, the class $A_{\{a, b\}, k, \text { loc }}^{p}$ is independent of the choice of $k \in(0,1)$ and we will denote it by $A_{\{a, b\}, \text { loc }}^{p}$.

\section{Preparatory Lemmas}

For a given $0<k<1, a \in \mathbb{R}^{d}, a=\left(a_{1}, a_{2}, \ldots, a_{d}\right)$, considering a grid of $\mathbb{R}^{d} \backslash\{a\}$ based on the sequence $\left\{ \pm k^{-s}\right\}_{s \in \mathbb{Z}}$, which results in the collection $\mathscr{G}_{a}$ of all the rectangles

$$
G_{a}=\left(I_{1}+\left\{a_{1}\right\}\right) \times\left(I_{2}+\left\{a_{2}\right\}\right) \times \cdots \times\left(I_{d}+\left\{a_{d}\right\}\right),
$$


where for a given $s \in \mathbb{Z}$, each $I_{j}$ is one of the intervals $\pm\left[k^{-s}, k^{-s-1}\right], \pm\left[0,((1-k) /(1+k)) k^{-s}\right]$, or $\pm[((1-k) /(1+$ $\left.k)) k^{-s}, k^{-s}\right]$ (called intervals of the first, second, or third type, resp.), but at least one of the intervals must be of the first type (if $0 \leq u<v<+\infty,-[u, v]$ denotes the interval $[-v,-u]$, while $+[u, v]$ is just $[u, v]$ ) If one of the intervals is of the second type, we say that $G_{a}$ is of the first category, otherwise of the second category.

For $G_{a} \in \mathscr{G}_{a}$, define $G_{a}^{\prime}$ to be the union of the cubes in $\mathcal{O}_{\{a\}, k}$ satisfying $Q \cap G_{a} \neq \emptyset$. If $G_{a}$ is of the first category, we define $G_{a}^{\prime \prime}$ to be the least cube containing $G_{a}^{\prime}$ with the largest distance from the point $a$ along each axis. If $G_{a}$ is of the second category, we define $G_{a, j}^{\prime \prime}$ to be the least cube containing $G_{a, j}^{\prime}=G_{a}^{\prime} \cap E_{j, G_{a}}$ with the largest distance from the point $a$ along each axis, where for every $j=1,2, \ldots, d$,

$$
E_{j, G_{a}}=\left\{\begin{array}{r}
\left\{y \in \mathbb{R}^{d}: y_{j} \geq \frac{1-k}{1+k} k^{-s}+a_{j}\right\}, \\
\text { when } I_{j} \subset(0,+\infty), \\
\left\{y \in \mathbb{R}^{d}: y_{j} \leq-\frac{1-k}{1+k} k^{-s}+a_{j}\right\}, \\
\text { when } I_{j} \subset(-\infty, 0) .
\end{array}\right.
$$

According to the definitions above, if $G_{a}$ is of the first category, we have $G_{a} \subset G_{a}^{\prime} \subset G_{a}^{\prime \prime}, G_{a}^{\prime} \subset\left\{y \in \mathbb{R}^{d}:\left|y_{j}-a_{j}\right| \geq\right.$ $\left.((1-k) /(1+k)) k^{-s}\right\}$ and $a \notin G_{a}^{\prime \prime}$. If $G_{a}$ is of the second category, then $G_{a} \subset G_{a, j}^{\prime}, a \notin G_{a, j}^{\prime \prime}, j=1,2, \ldots, d$ and $G_{a}^{\prime} \cap \prod_{j=1}^{d} E_{j, G_{a}}^{c}=\emptyset$ which equals $G_{a}^{\prime}=\bigcup_{j=1}^{d} G_{a, j}^{\prime}$.

For a point $b \in \mathbb{R}^{d}$, repeat the process above, we can obtain the corresponding sets $G_{b}, G_{b}^{\prime}, G_{b}^{\prime \prime}, G_{b, j}^{\prime}, G_{b, j}^{\prime \prime}$, and $\mathscr{G}_{b}$.

For $a, b \in \mathbb{R}^{d}(a \neq b)$,

$$
\begin{aligned}
\mathbb{R}^{d} \backslash\{a, b\} & =\left(\bigcup_{G_{a} \in \mathscr{G}_{a}} G_{a}\right) \cap\left(\bigcup_{G_{b} \in \mathscr{G}_{b}} G_{b}\right) \\
& =\bigcup_{G_{a} \in \mathscr{G}_{a}, G_{b} \in \mathscr{G}_{b}}\left(G_{a} \cap G_{b}\right) .
\end{aligned}
$$

Define $\mathscr{G}_{a, b}$ to be the collection of $G_{a, b}=G_{a} \cap G_{b}$, where $G_{a} \in \mathscr{G}_{a}$ and $G_{b} \in \mathscr{G}_{b}$ and $G_{a, b}^{\prime}$ to be the union of the cubes $Q \in \mathcal{O}_{\{a, b\}, k}$ such that $Q \cap G_{a, b} \neq \emptyset$. In the proof of Theorem 6, we will use a result; that is, $G_{a, b}^{\prime} \subset\left(G_{a}^{\prime} \cap G_{b}^{\prime}\right)$.

In fact, for every $z \in G_{a, b}^{\prime}$, there exists a cube $Q_{0} \in \mathcal{O}_{\{a, b\}, k}$, $Q_{0} \cap G_{a, b} \neq \emptyset$ such that $z \in Q_{0}$. The definition of $\mathcal{O}_{\{a, b\}, k}$ gives that there exists a $y \in \mathbb{R}^{d} \backslash\{a, b\}$ such that $Q_{0}$ is the cube centered at $y$ with radius $r \leq k\|y-a\|$. Then $Q_{0} \in \mathcal{O}_{\{a\}, k}$ and $Q_{0} \subset G_{a}^{\prime}$; consequently $z \in G_{a}^{\prime}$. Similarly, $z \in G_{b}^{\prime}$. Thus $z \in G_{a}^{\prime} \cap G_{b}^{\prime}$.

Lemma 4. Given $0<k<1$, there exists $k_{0}=k_{0}(d, k) \in(0,1)$ such that for each $G_{a, b}=G_{a} \cap G_{b} \in \mathscr{G}_{a, b}, G_{a}^{\prime \prime} \in \mathcal{O}_{\{a\}, k_{0}}$ (or $\left.G_{b}^{\prime \prime} \in \mathcal{O}_{\{b\}, k_{0}}\right)$ if $G_{a}\left(\right.$ or $\left.G_{b}\right)$ is of the first category, and $G_{a, j}^{\prime \prime} \in$ $\mathcal{O}_{\{a\}, k_{0}}\left(\right.$ or $\left.G_{b, j}^{\prime \prime} \in \mathcal{O}_{\{b\}, k_{0}}\right), j=1,2, \ldots, d$, if $G_{a}\left(\right.$ or $\left.G_{b}\right)$ is of the second category.
Proof. The constants $k_{a}$ and $k_{b}$ associated with the points $a$ and $b$, respectively, can be obtained by the same procedure as the one in the proof of Lemma 3.1 in [4]. Then we have $G_{a}^{\prime \prime} \in \mathcal{O}_{\{a\}, k_{a}}$ and $G_{b}^{\prime \prime} \in \mathcal{O}_{\{b\}, k_{b}}$ when $G_{a}$ and $G_{b}$ are of the first category. Let $k_{0}=\max \left\{k_{a}, k_{b}\right\}$, thus $G_{a}^{\prime \prime} \in \mathcal{O}_{\{a\}, k_{0}}$ and $G_{b}^{\prime \prime} \in \mathcal{O}_{\{b\}, k_{0}}$ by the definition of $\mathcal{O}_{\{a\}, k_{0}}$ and $\mathcal{O}_{\{b\}, k_{0}}$. The case when $G_{a}$ and $G_{b}$ are of the second category is similar to the case when $G_{a}$ and $G_{b}$ are of the first category.

Lemma 5. Given $0<k<1$. Let $Q$ and $\widetilde{Q}$ be the cubes in $\mathbb{R}^{d}$ satisfying $Q \subset \widetilde{Q}$. If $\widetilde{Q} \in \mathcal{O}_{\{a\}, k}\left(\right.$ or $\left.\mathcal{O}_{\{b\}, k}\right)$; then $Q \in$ $\mathcal{O}_{\{a\}, k}\left(\operatorname{or} \mathcal{O}_{\{b\}, k}\right)$.

Lemma 5 can be obtained from Lemma 3.3 in [4] by translating the cubes in it.

\section{The Main Results}

Now we come to the main results of the paper.

Theorem 6. Let $0<k<1$ and $1 \leq p<\infty$. If $w \in A_{\{a, b\}, \mathrm{loc}}^{p}$ then the double points local maximal operator $M_{\{a, b\}, k, \text { loc }}$ is bounded on $L^{p}(w)$ when $p>1$ and bounded from $L^{1}(w)$ into $L^{1, \infty}(w)$. Moreover, the corresponding constant depends only on the double points local $k_{0}-A_{p}$ constant of $w$ for some $k_{0}$, $0<k_{0}<1$.

Proof. Denote $M_{\{a, b\}, k, \text { loc }}$ by $M_{k \text {,loc }}$ for short. The definition of $G_{a, b}^{\prime}$ gives that $M_{k, \text { loc }} f(x)=M_{k, \text { loc }}\left(f \chi_{G_{a, b}^{\prime}}\right)(x)$ for $x \in G_{a, b}$. First, we consider the case $p>1$

$$
\begin{aligned}
\int_{\mathbb{R}^{d}} & \left|M_{k, \text { loc }} f(x)\right|^{p} w(x) d x \\
& =\sum_{G_{a, b} \in \mathscr{E}_{a, b}} \int_{G_{a, b}}\left|M_{k, \mathrm{loc}} f(x)\right|^{p} w(x) d x \\
& =\sum_{G_{a, b} \in \mathscr{E}_{a, b}} \int_{G_{a, b}}\left|M_{k, \mathrm{loc}}\left(f \chi_{G_{a, b}^{\prime}}\right)(x)\right|^{p} w(x) d x \\
& \leq \sum_{G_{a, b} \in \mathscr{E}_{a, b}} \int_{G_{a, b}}\left|M\left(f \chi_{G_{a, b}^{\prime}}\right)(x)\right|^{p} w(x) d x .
\end{aligned}
$$

Since $M$ is sublinear and $G_{a, b}^{\prime} \subset\left(G_{a}^{\prime} \cap G_{b}^{\prime}\right)$, we have

$$
\begin{aligned}
& \int_{\mathbb{R}^{d}}\left|M_{k, \mathrm{loc}} f(x)\right|^{p} w(x) d x \\
& \leq 2^{p} \sum_{G_{a, b} \in \mathscr{G}_{a, b}}\left(\int_{G_{a, b}}\left|M\left(f \chi_{G_{a}^{\prime}}\right)(x)\right|^{p} w(x) d x\right. \\
&\left.\quad+\int_{G_{a, b}}\left|M\left(f \chi_{G_{b}^{\prime}}\right)(x)\right|^{p} w(x) d x\right)
\end{aligned}
$$




$$
\begin{aligned}
& =2^{p}\left(\int_{\mathbb{R}^{d}}\left|M\left(f \chi_{G_{a}^{\prime}}\right)(x)\right|^{\left.\right|^{p}} w(x) d x\right. \\
& \left.+\int_{\mathbb{R}^{d}}\left|M\left(f \chi_{G_{b}^{\prime}}\right)(x)\right|^{p} w(x) d x\right) \\
& =2^{p}\left(\sum_{G_{a} \in \mathscr{G}_{a}} \int_{G_{a}}\left|M\left(f \chi_{G_{a}^{\prime}}\right)(x)\right|^{p} w(x) d x\right. \\
& \left.\quad+\sum_{G_{b} \in \mathscr{G}_{b}} \int_{G_{b}}\left|M\left(f \chi_{G_{b}^{\prime}}\right)(x)\right|^{p} w(x) d x\right) .
\end{aligned}
$$

Translating the cubes along $a$ and $b$, respectively, in the corresponding part of proof for Theorem 4.1 in [4] gives

$$
\begin{aligned}
& \int_{G_{a}}\left|M\left(f \chi_{G_{a}^{\prime}}\right)(x)\right|^{p} w(x) d x \\
& \leq C\|w\|_{A_{\{a\}, k_{0}, \mathrm{loc}}^{p}}^{p p^{\prime}} \int_{G_{a}^{\prime}}|f(x)|^{p} w(x) d x, \\
& \int_{G_{b}} \mid\left.M\left(f \chi_{G_{b}^{\prime}}\right)(x)\right|^{p} w(x) d x \\
& \leq C\|w\|_{A_{\{b\}, k_{0}, \text { loc }}^{p p^{\prime}}} \int_{G_{b}^{\prime}}|f(x)|^{p} w(x) d x .
\end{aligned}
$$

Note that the families $\left\{G_{a}^{\prime}\right\}_{G_{a} \in \mathscr{G}_{a}}$ and $\left\{G_{b}^{\prime}\right\}_{G_{b} \in \mathscr{G}_{b}}$ have the finite overlapping property; that is, there exists a constant $m=$ $m(d, k)$ such that every $x \in \mathbb{R}^{d} \backslash\{a, b\}$ belongs to at most $m$ sets from $\left\{G_{a}^{\prime}\right\}_{G_{a} \in \mathscr{G}_{a}}$ or $\left\{G_{b}^{\prime}\right\}_{G_{a} \in \mathscr{G}_{b}}$. Combining this with (16) gives

$$
\begin{aligned}
& \int_{\mathbb{R}^{d}} \mid\left.M_{k, \text { loc }} f(x)\right|^{p} w(x) d x \\
& \leq 2^{p}\left(\sum_{G_{a} \in \mathscr{G}_{a}} C\|w\|_{A_{\{a\}, k_{0}, \text { loc }}^{p}}^{p p^{\prime}} \int_{G_{a}^{\prime}}|f(x)|^{p} w(x) d x\right. \\
&\left.+\sum_{G_{b} \in \mathscr{G}_{b}} C\|w\|_{A_{\{b\}, k_{0}, \mathrm{loc}}^{p}}^{p p_{G_{b}^{\prime}}^{\prime}}|f(x)|^{p} w(x) d x\right) \\
& \leq C B_{k_{0}, p}\left(\int_{\mathbb{R}^{d}}|f(x)|^{p}\left(\sum_{G_{a} \in \mathscr{G}_{a}} \chi_{G_{a}^{\prime}}\right) w(x) d x\right.\left.+\int_{\mathbb{R}^{d}}|f(x)|^{p}\left(\sum_{G_{b} \in \mathscr{G}_{b}} \chi_{G_{b}^{\prime}}\right) w(x) d x\right) \\
& \leq C m B_{k_{0}, p} \int_{\mathbb{R}^{d}}|f(x)|^{p} w(x) d x,
\end{aligned}
$$

where $B_{k_{0}, p}=\max \left\{\|w\|_{A_{\{a\}, k_{0}, \text { loc }}^{p}}^{p p^{\prime}},\|w\|_{A_{\{b\}, k_{0}, \text { loc }}^{p}}^{p p^{\prime}}\right\}$.
As for the case $p=1$, we define the level sets

$$
\begin{gathered}
E_{\lambda}=\left\{x \in \mathbb{R}^{d}: M_{k, \mathrm{loc}} f(x)>\lambda\right\}, \\
E_{\lambda, G_{a, b}^{\prime}}=\left\{x \in \mathbb{R}^{d}: M_{k, \mathrm{loc}}\left(f \chi_{G_{a, b}^{\prime}}\right)(x)>\lambda\right\}, \\
F_{\lambda, G_{a, b}^{\prime}}=\left\{x \in \mathbb{R}^{d}: M\left(f \chi_{G_{a, b}^{\prime}}\right)(x)>\lambda\right\}, \\
F_{\lambda, G_{a}^{\prime}}=\left\{x \in \mathbb{R}^{d}: M\left(f \chi_{G_{a}^{\prime}}\right)(x)>\lambda\right\}, \\
F_{\lambda, G_{b}^{\prime}}=\left\{x \in \mathbb{R}^{d}: M\left(f \chi_{G_{b}^{\prime}}\right)(x)>\lambda\right\} .
\end{gathered}
$$

Since $F_{\lambda, G_{a, b}^{\prime}} \subset\left(F_{\lambda, G_{a}^{\prime}} \cup F_{\lambda, G_{b}^{\prime}}\right)$, the weak weighed boundedness of the maximal operator $M$ and the similar arguments as the case $p>1$ give that

$$
\begin{aligned}
\int_{E_{\lambda}} w(x) d x= & \sum_{G_{a, b} \in \mathscr{G}_{a, b}} \int_{G_{a, b}} \chi_{E_{\lambda, G_{a, b}^{\prime}}}(x) w(x) d x \\
\leq & \sum_{G_{a, b} \in \mathscr{G}_{a, b}} \int_{G_{a, b}} \chi_{F_{\lambda, G_{a, b}^{\prime}}}(x) w(x) d x \\
\leq & \sum_{G_{a} \in \mathscr{G}_{a}} \int_{G_{a}} \chi_{F_{\lambda, G_{a}^{\prime}}}(x) w(x) d x \\
& +\sum_{G_{b} \in \mathscr{G}_{b}} \int_{G_{b}} \chi_{F_{\lambda, G_{b}^{\prime}}}(x) w(x) \mathrm{d} x \\
\leq & C B_{k_{0}, 1} \lambda^{-1}\left(\sum_{G_{a} \in \mathscr{G}_{a}} \int_{G_{a}^{\prime}}|f(x)| w(x) d x\right. \\
& \left.+\sum_{G_{b} \in \mathscr{G}_{b}} \int_{G_{b}^{\prime}}|f(x)| w(x) d x\right) \\
\leq & C m B_{k_{0}, 1} \lambda^{-1} \int_{\mathbb{R}^{d}}|f(x)| w(x) d x,
\end{aligned}
$$

where $B_{k_{0}, 1}=\max \left\{\|w\|_{A_{\{a\}, k_{0}, \text { loc }}^{1}},\|w\|_{A_{\{b\}, k_{0}, \text { loc }}^{1}}\right\}$. Thus the proof is finished.

Theorem 7. Let $0<k<1$ and $1 \leq p<\infty$, w be a weight function on $\mathbb{R}^{d}$. The condition $w \in A_{\{a, b\}, \text { loc }}^{p}$ is necessary for the double points local maximal operator $M_{\{a, b\}, k, \text { loc }}$ to be bounded from $L^{p}(w)$ into $L^{p, \infty}(w)$; that is,

$$
\begin{aligned}
w(\{x & \left.\left.\in \mathbb{R}^{d}: M_{\{a, b\}, k, \text { loc }} f(x)>\lambda\right\}\right) \\
& \leq \frac{C}{\lambda^{p}} \int_{\mathbb{R}^{d}}|f(x)|^{p} w(x) d x, \quad \lambda>0 .
\end{aligned}
$$

The proof of Theorem 7 is analogous to that of Theorem 4.2 in [4], and we omit it here.

\section{Acknowledgments}

This paper is supported by Project of Science and Technology Research Foundation of Educational Commission 
of Heilongjiang Province (12511158), Foundation for Young Key Teacher of Harbin Normal University (KGB201011), and Natural Science Foundation of Heilongjiang Province (A201206).

\section{References}

[1] W. Li, L. Xue, and X. Yan, "Sharp maximal function estimate and weighted inequalities for maximal multilinear singular integrals," Analysis in Theory and Applications, vol. 27, no. 2, pp. 158-166, 2011.

[2] A. K. Lerner, "Some remarks on the Hardy-Littlewood maximal function on variable $L^{p}$ spaces," Mathematische Zeitschrift, vol. 251, no. 3, pp. 509-521, 2005.

[3] D. Gallardo, "Weighted weak type integral inequalities for the Hardy-Littlewood maximal operator," Israel Journal of Mathematics, vol. 67, no. 1, pp. 95-108, 1989.

[4] C.-C. Lin and K. Stempak, "Local Hardy-Littlewood maximal operator," Mathematische Annalen, vol. 348, no. 4, pp. 797-813, 2010.

[5] B. Muckenhoupt, "Weighted norm inequalities for the Hardy maximal function," Transactions of the American Mathematical Society, vol. 165, pp. 207-226, 1972.

[6] S. M. Buckley, "Estimates for operator norms on weighted spaces and reverse Jensen inequalities," Transactions of the American Mathematical Society, vol. 340, no. 1, pp. 253-272, 1993. 


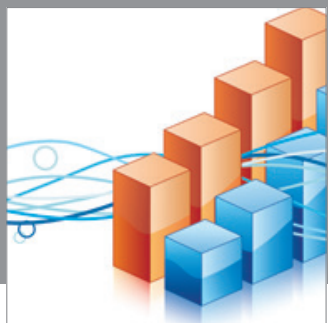

Advances in

Operations Research

mansans

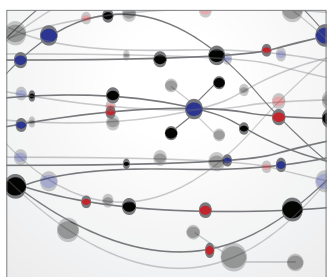

The Scientific World Journal
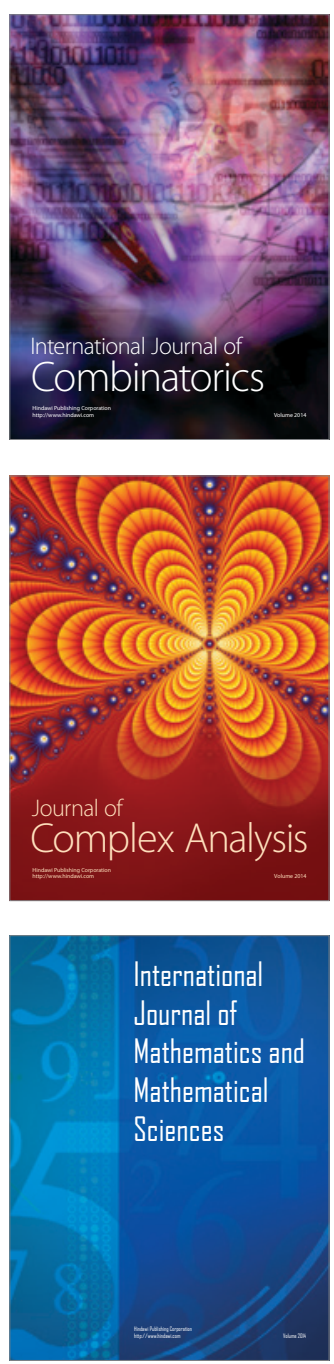
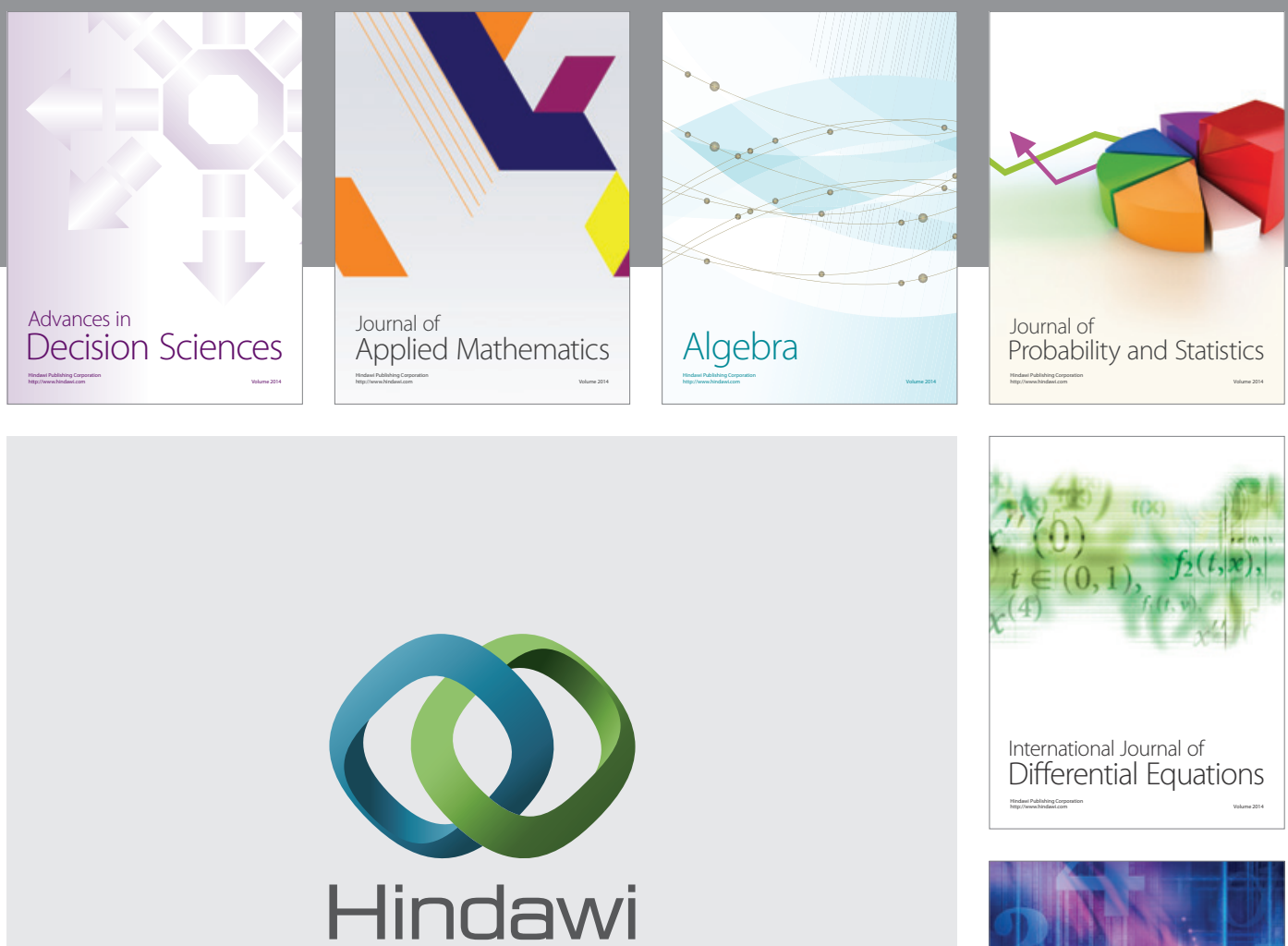

Submit your manuscripts at http://www.hindawi.com
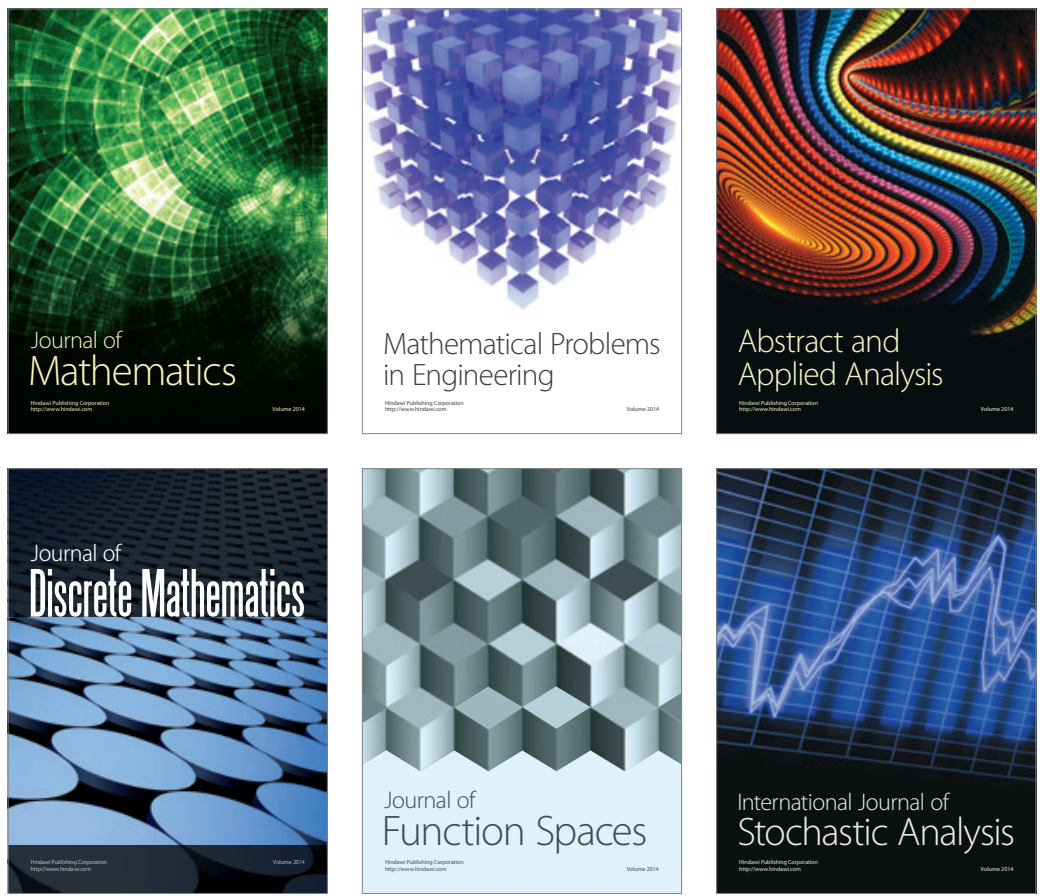

Journal of

Function Spaces

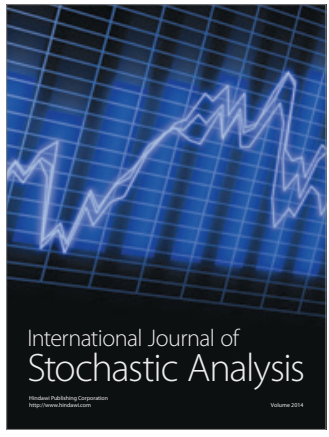

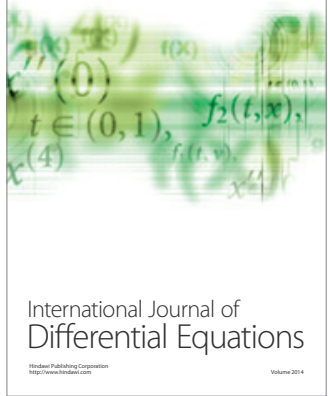
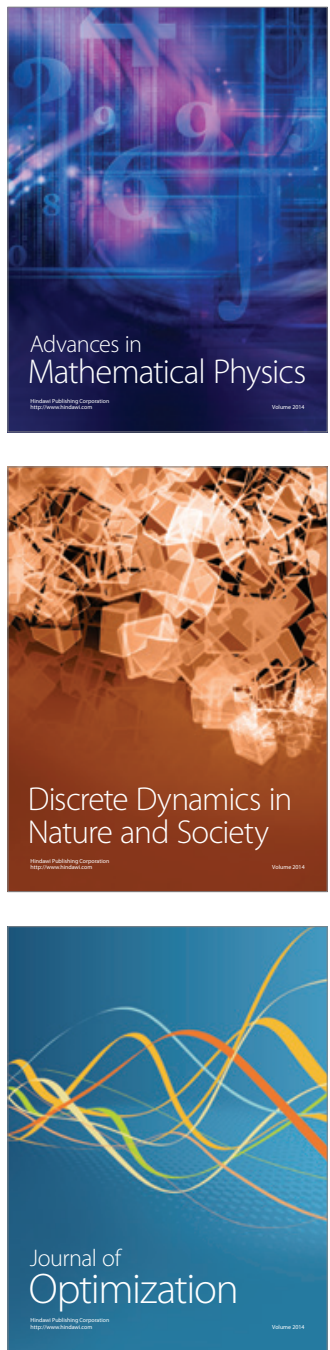